\title{
Measurements of diffractive and exclusive processes with ATLAS
}

\section{Mateusz Dyndal (DESY)}

on behalf of the ATLAS Collaboration

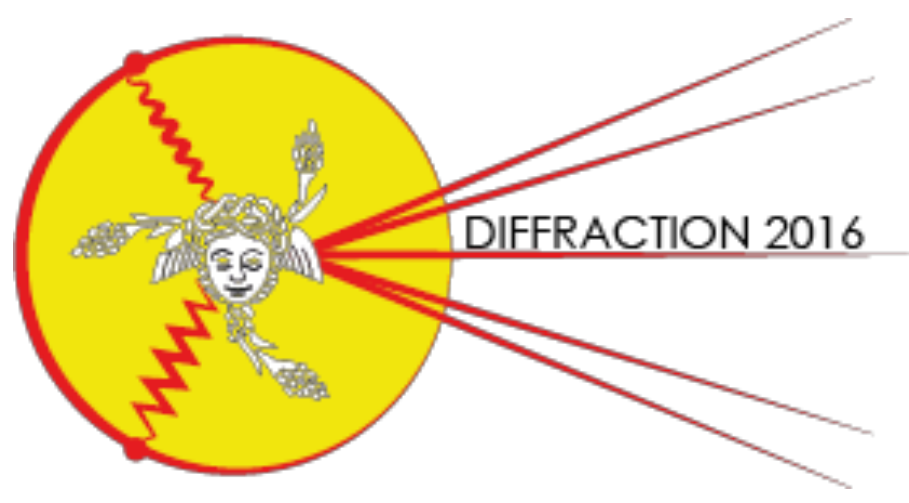

2-8 Sep 2016 


\section{Outline}

- Exclusive $\gamma \gamma \rightarrow \ell^{+} \ell^{-}$Production at $7 \mathrm{TeV}$

- Exclusive $\gamma \gamma \rightarrow \mathrm{W}^{+} \mathrm{W}^{-}$Production and Search for Exclusive Higgs Production at $8 \mathrm{TeV}$

- Diffractive Dijet Cross Sections at $7 \mathrm{TeV}$

- Feasibility Studies for Exclusive Jet Production with AFP 


\section{Exclusive photon-induced processes: Motivation}

- Exclusive $\gamma \gamma \rightarrow X$ production can be computed in QED+EWK with relatively small uncertainty (EPA)

- True if we neglect proton absorptive corrections...

- Exclusive $\gamma \gamma \rightarrow \ell^{+} \ell^{-}$production

- Standard candle for photon-induced physics

- Non-negligible background to Drell-Yan like reactions

- Possible to use pp $(\gamma \gamma) \rightarrow p p \ell^{+} \ell^{-}$for luminosity calibration at the LHC?

- Exclusive $\mathrm{W}^{+} \mathrm{W}^{-}$

- Test of SM $y$ WWW quartic gauge coupling

- Probe of anomalous quartic gauge couplings (aQGCs)

- Exclusive (CEP) gg $\rightarrow$ Higgs $\rightarrow \mathrm{W}^{+} \mathrm{W}^{-}$

- Similar final state as in exclusive $\mathrm{y} \gamma \rightarrow \mathrm{W}^{+} \mathrm{W}^{-}$studies

- Can be used for Higgs properties studies (low systematics due to the clean production environment) 


\section{Exclusive $y y \rightarrow \ell^{+} \ell^{-}$production at $7 \mathrm{TeV}$, PLB 749 (2015) 242-261}

- Photon-induced processes: cross-section dominated by so-called single- and double-proton dissociative reactions. Non-negligible background for many analyses (low, high-mass DY, $\phi^{*} / \mathrm{p}_{\mathrm{T}}(\mathrm{Z})$ measurement, ...)

- Preselection:

- $p_{\mathrm{T}}{ }^{\mu}>10 \mathrm{GeV},\left|\eta_{\mu}\right|<2.4, \mathrm{M}_{\mu+\mu-}>20 \mathrm{GeV}$

- $p_{\mathrm{T}}{ }^{\mathrm{e}}>12 \mathrm{GeV},\left|\eta_{\mathrm{e}}\right|<2.4, \mathrm{M}_{\mathrm{e}+\mathrm{e}}>24 \mathrm{GeV}$
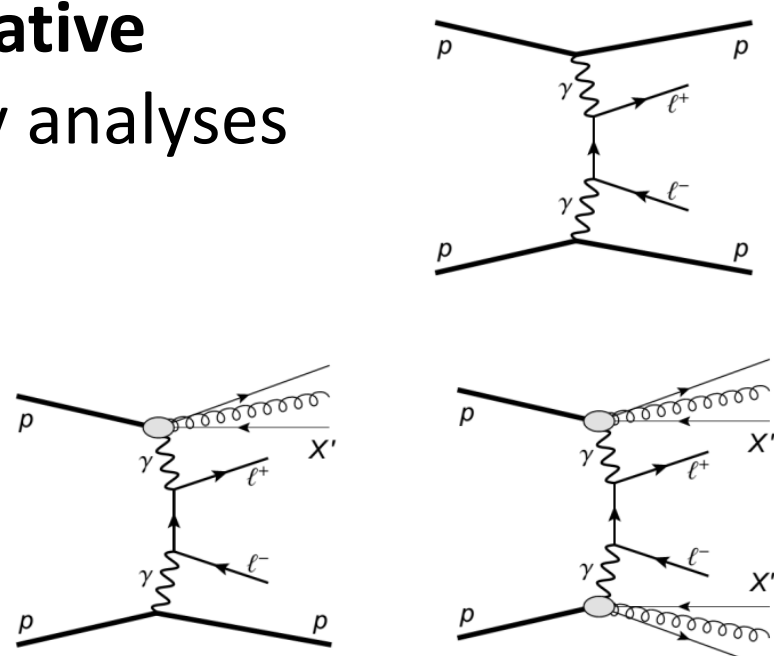

- Exclusive selection:

- 3 mm dilepton-vertex longitudinal isolation efficiency $=\mathbf{7 4 \%}$

- $\boldsymbol{p}_{\mathrm{T}}$ of the dilepton system $<1.5 \mathrm{GeV}$
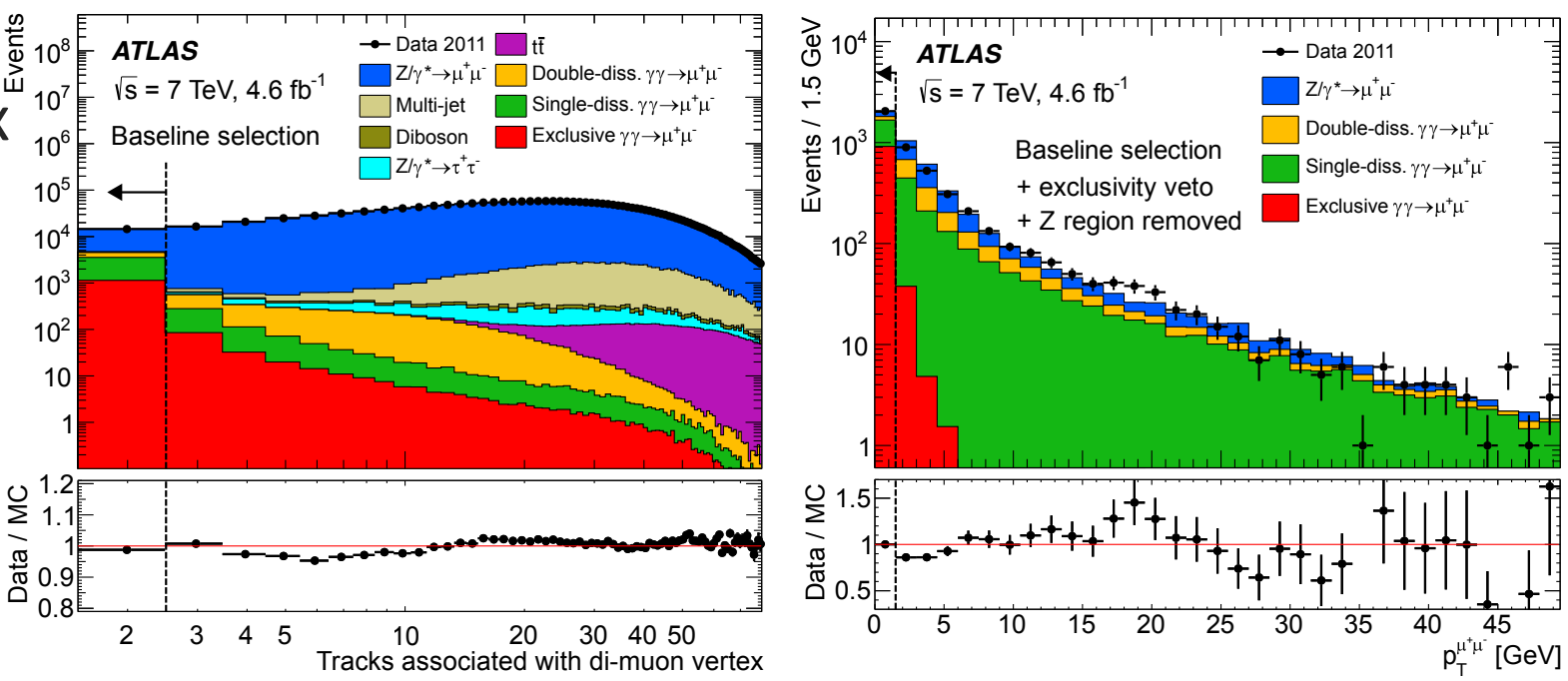


\section{Exclusive $y \gamma \rightarrow \ell^{+} \ell^{-}$production at $7 \mathrm{TeV}$, PLB 749 (2015) 242-261}

- Signal extraction: binned maximum-likelihood fit to the measured dilepton acoplanarity distribution

- Corresponding fiducial cross-sections:

- $\sigma_{\gamma \gamma \rightarrow e+e_{-}}^{\text {excl. }}=0.428 \pm 0.035$ (stat.) \pm 0.018 (syst.) pb

- $\sigma_{\gamma \gamma \rightarrow \mu+\mu-}^{\text {excl. }}=0.628 \pm 0.032$ (stat.) \pm 0.021 (syst.) pb

- Theory predictions (QED-EPA), with absorptive

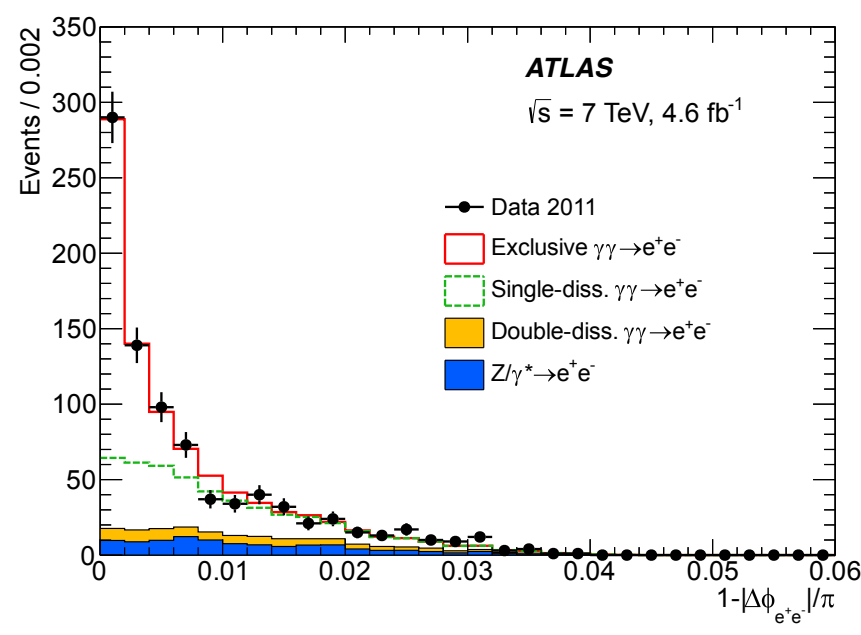
corrections from PLB 741 (2015) 66-70 (20\% effect)

- $\sigma_{\gamma \gamma \rightarrow e+e-}^{E P A, \text { corr. }}=0.398 \pm 0.007$ (theo.) pb

- $\sigma_{\gamma \gamma \rightarrow \mu+\mu-}^{E P A, \text { corr. }}=0.638 \pm 0.011$ (theo.) pb

- Agreement also with similar CMS measurement

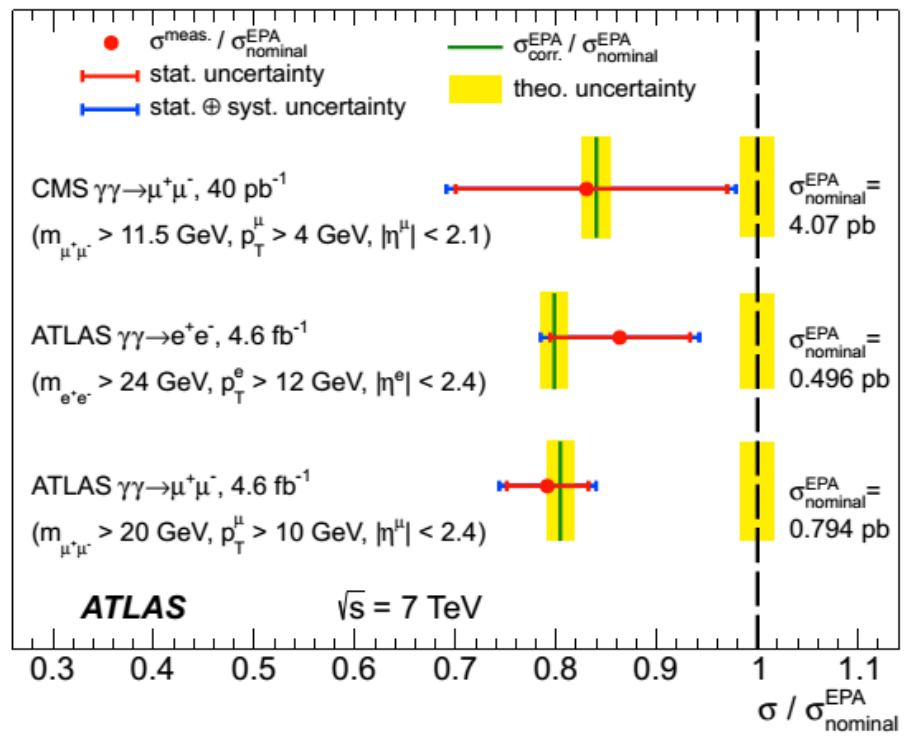




\section{Exclusive $\gamma \psi \rightarrow W^{+} W^{-}$and Search for Exclusive $H$ at $8 \mathrm{TeV}$}

(arXiv:1607.03745) PRD 94 (2016) 032011

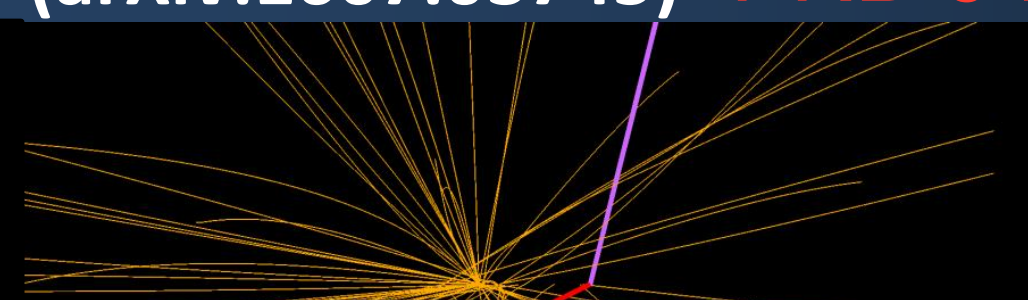

\section{ATLAS L I EXPERIMENT http://atlas.ch}

Run: 203432

Event: 53911100

2012-05-15 13:35:15 CEST

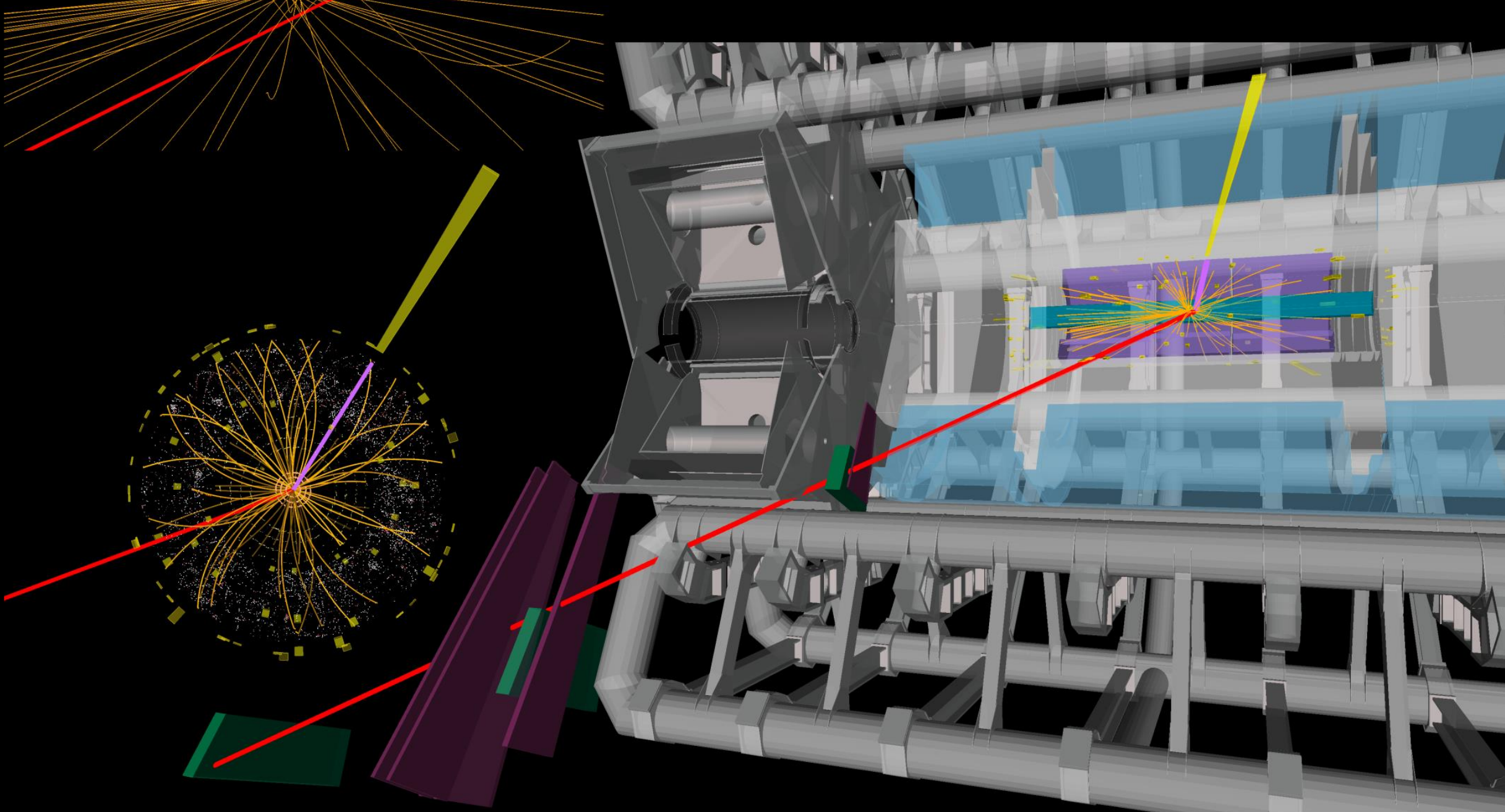




\section{Exclusive $\gamma \gamma \rightarrow W^{+} W^{-}$and Search for Exclusive $H$ at $8 \mathrm{TeV}$ (arXiv:1607.03745)}

- Event selection

- WW $\rightarrow$ ev $\mu v$ final states are considered

- $1 \mathrm{~mm}$ dilepton-vertex longitudinal isolation $->$ efficiency $=58 \pm 6 \%$

- Full event selection criteria:

\begin{tabular}{|c|c|c|}
\hline Variable & Excl $W^{+} W^{-}$ & Excl Higgs \\
\hline$p_{T}^{\text {lep }}$ & $>25,20 \mathrm{GeV}$ & $>25,15 \mathrm{GeV}$ \\
$m_{e \mu}$ & $>20 \mathrm{GeV}$ & $>10 \mathrm{GeV}$ \\
$p_{T}^{e \mu}$ & $>30 \mathrm{GeV}$ & $>30 \mathrm{GeV}$ \\
$\Delta z_{0}^{i s o}$ & $1 \mathrm{~mm}$ & $1 \mathrm{~mm}$ \\
\hline$p_{T}^{e \mu}(\mathrm{aQGC})$ & $>120 \mathrm{GeV}$ & - \\
\hline$m_{e \mu}$ & - & $<55 \mathrm{GeV}$ \\
$\Delta \phi_{e \mu}$ & - & $<1.8$ \\
$m_{T}$ & - & $<140 \mathrm{GeV}$ \\
\hline
\end{tabular}

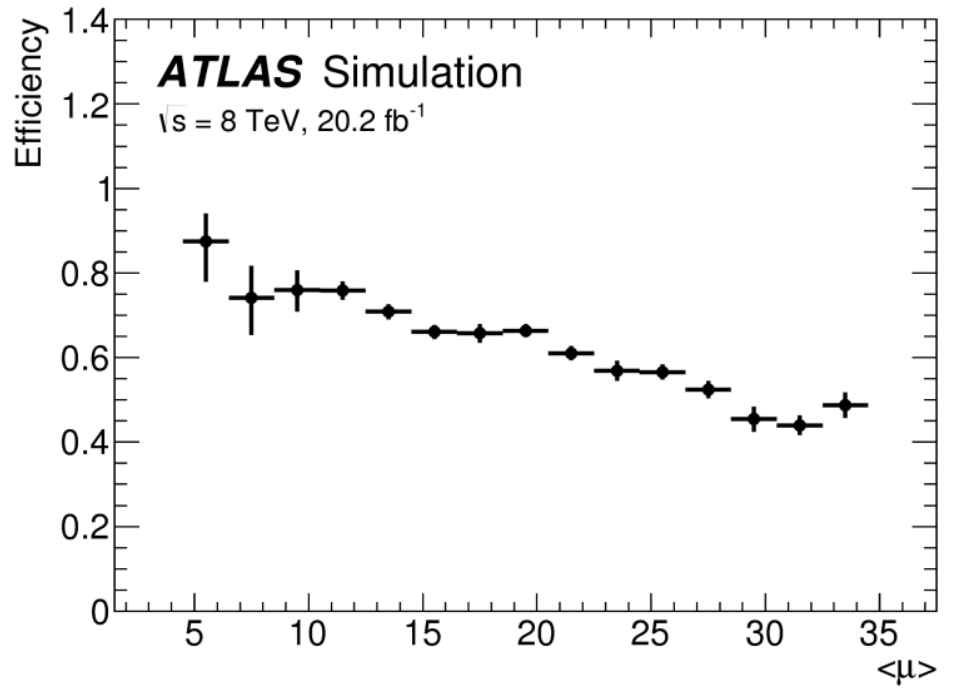

Higgs selection: lower pT / mass requirement (one $\mathrm{W}$ is off-shell) 


\section{Exclusive $\gamma \psi \rightarrow W^{+} W^{-}$and Search for Exclusive $H$ at $8 \mathrm{TeV}$ (arXiv:1607.03745)}

- $\gamma \gamma \rightarrow \ell^{+} \ell^{-}$validation

- Ratio of observed elastic $\boldsymbol{\gamma} \boldsymbol{\gamma} \rightarrow \ell^{+} \ell^{-}$to bare EPA prediction:

$f_{\mathrm{EL}}=0.76 \pm 0.04$ (stat.) \pm 0.10 (sys.)

-> Suppression is stronger due to larger invariant mass being probed

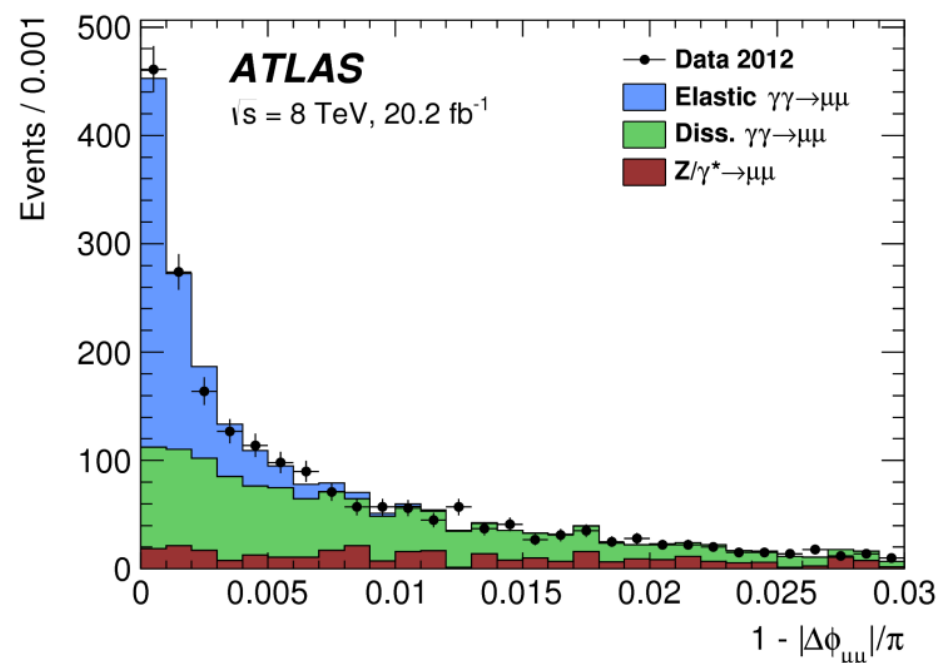

- No simulation available for SD and DD $\gamma p \rightarrow \mathrm{W}^{+} \mathrm{W}^{-}$ (and EL+SD+DD are mixed due to $W$ decays): a correction factor is applied using $\boldsymbol{\gamma \boldsymbol { \gamma }} \rightarrow \ell^{+} \ell^{-}$ for $\mathrm{m}_{\ell+\ell-}>160 \mathrm{GeV}$ :

$f_{\gamma}=\left.\frac{N_{\text {Data }}-N_{\text {Background }}^{\text {PowHEG }}}{N_{\text {Elastic }}^{\text {HERIG+ }}}\right|_{m_{\mu \mu}>160 \mathrm{GeV}}=3.30 \pm 0.22$ (stat.) \pm 0.06 (sys.)

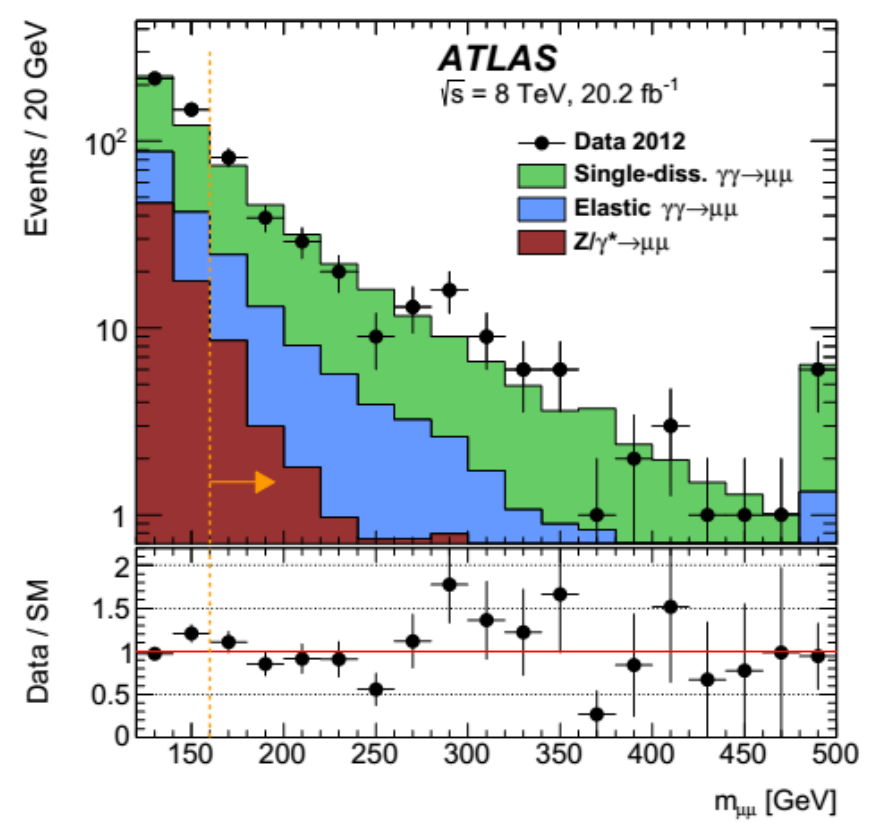




\section{Exclusive $\gamma \gamma \rightarrow W^{+} W^{-}$and Search for Exclusive $H$ at $8 \mathrm{TeV}$ (arXiv:1607.03745)}

- Results ( $\gamma \gamma \rightarrow \mathbf{W}^{+} \mathbf{W}^{-}$and aQGCs)

- Exclusive $\mathrm{W}^{+} \mathrm{W}^{-}$event yields: Data $=23$, Background $=8.3 \pm 2.6$, Signal $=9.3 \pm 1.2$ ->Measurement significance of $3 \sigma$

- aQGC event yields [pT(e $\mu)>120 \mathrm{GeV}]$ : Data $=1$, Background $=0.37 \pm 0.13$, SM Signal $=0.37 \pm 0.04$ -> new aQGC limits are set

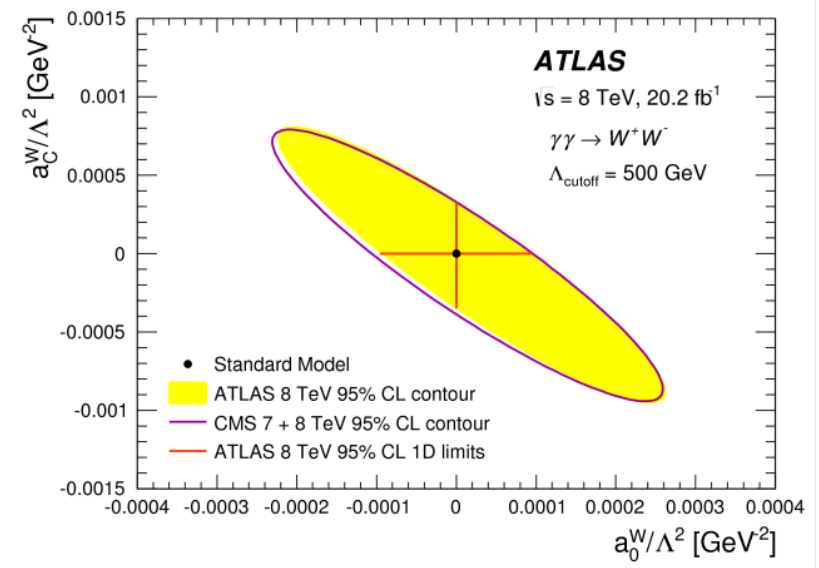

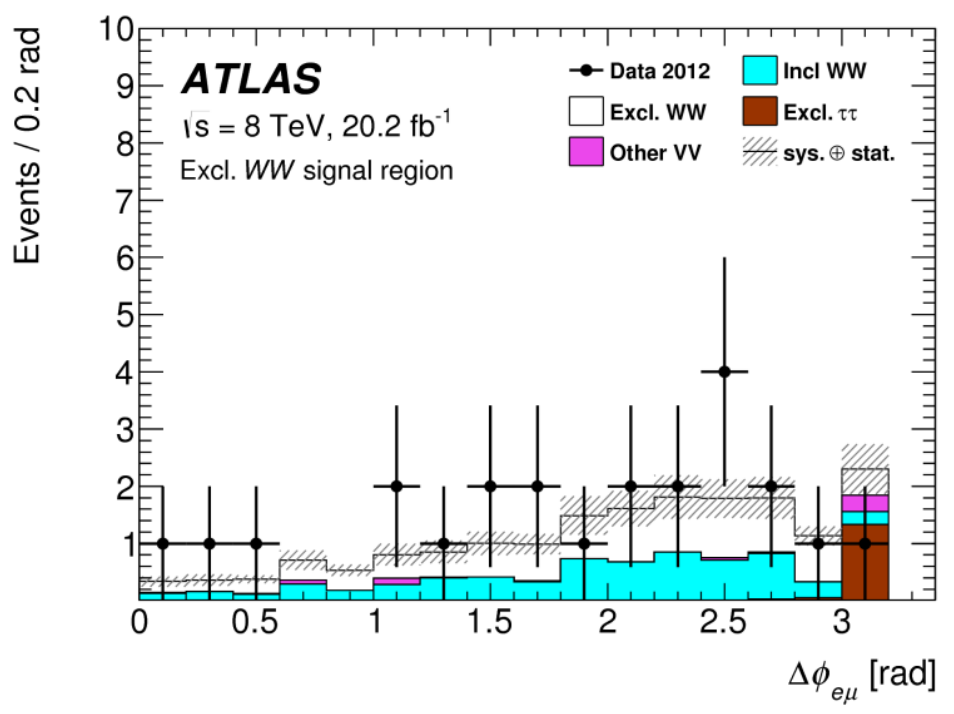

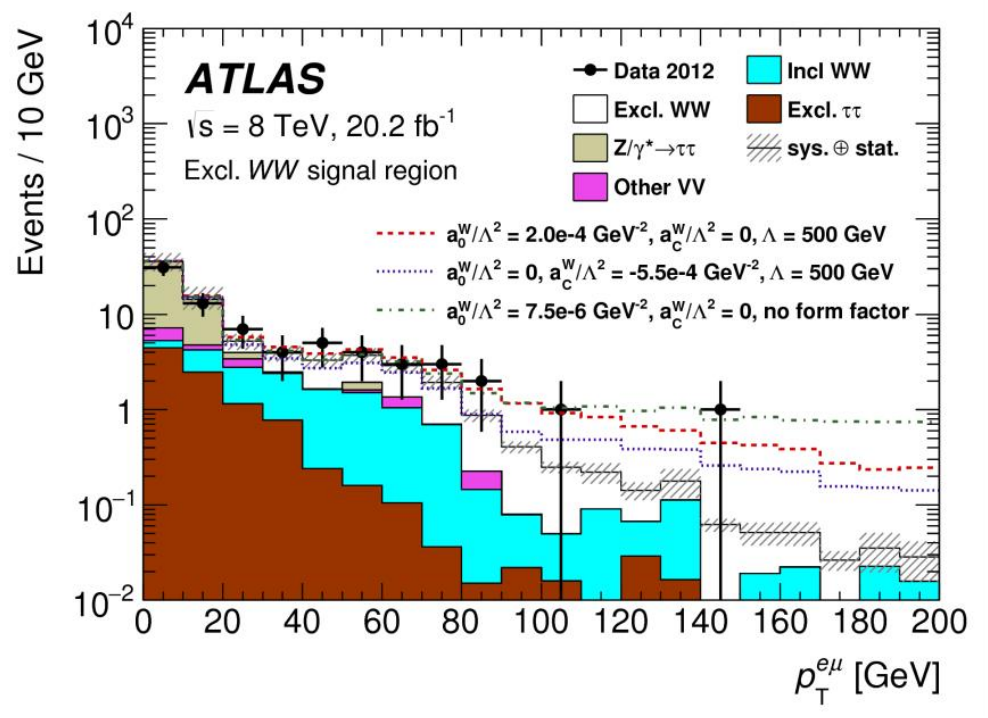




\section{Exclusive $\gamma \gamma \rightarrow W^{+} W^{-}$and Search for Exclusive $H$ at $8 \mathrm{TeV}$ (arXiv:1607.03745)}

- Results (exclusive Higgs)

- Exclusive and inclusive $\mathrm{W}^{+} \mathrm{W}^{-}$are the dominant background

- Exclusive Higgs event yields: Data=6, Background $=3.0 \pm 0.8$, Signal $=0.023 \pm 0.003$

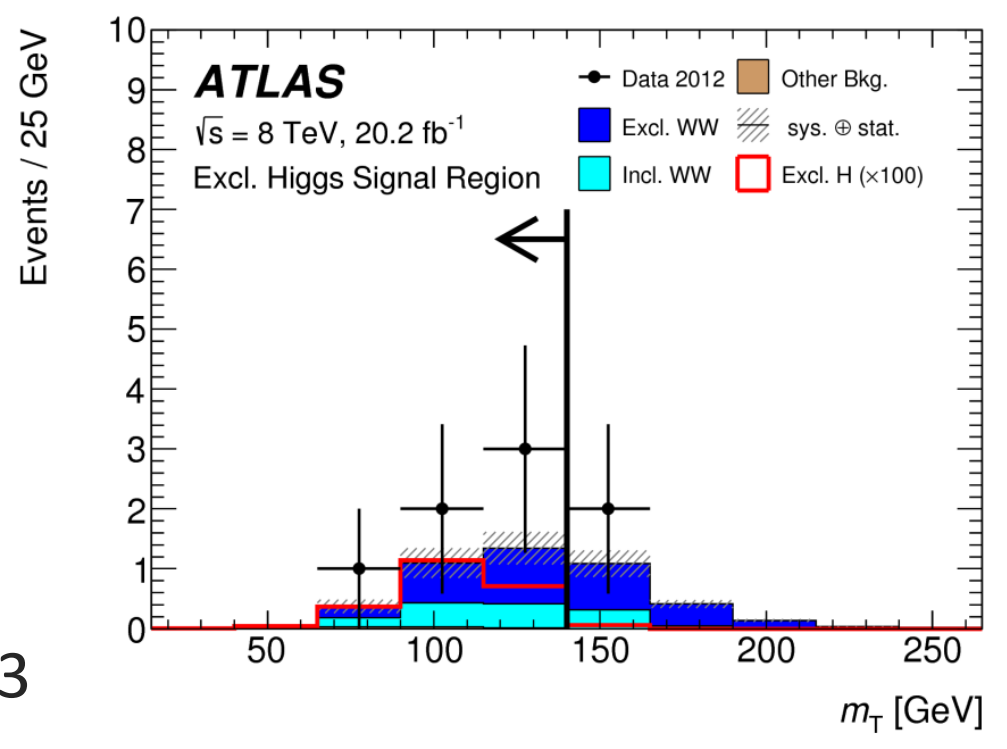

- Observed and expected limits: $\sigma<1.2$ pb @ 95\% CL (Observed) $\sigma<0.7 \mathrm{pb} @ 95 \% \mathrm{CL}$ (Expected)

- Upper limit $=400 \times$ predicted $\sigma$ (predictions include just the elastic process)

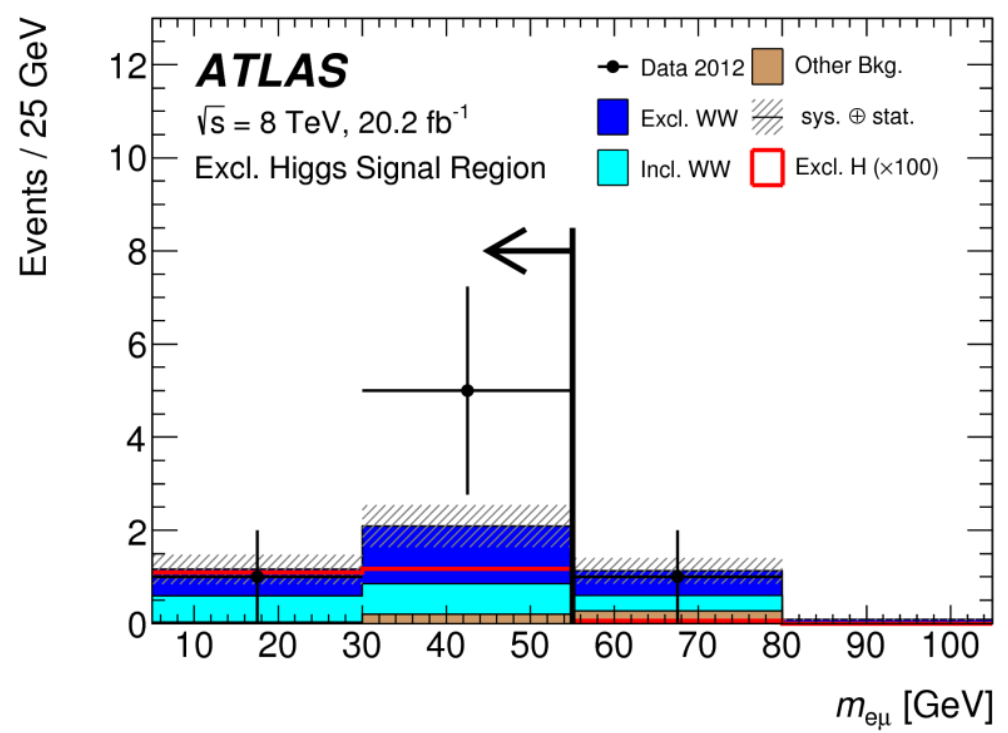




\section{Diffractive Dijet Production at 7 TeV, PLB 754 (2016) 214-234}

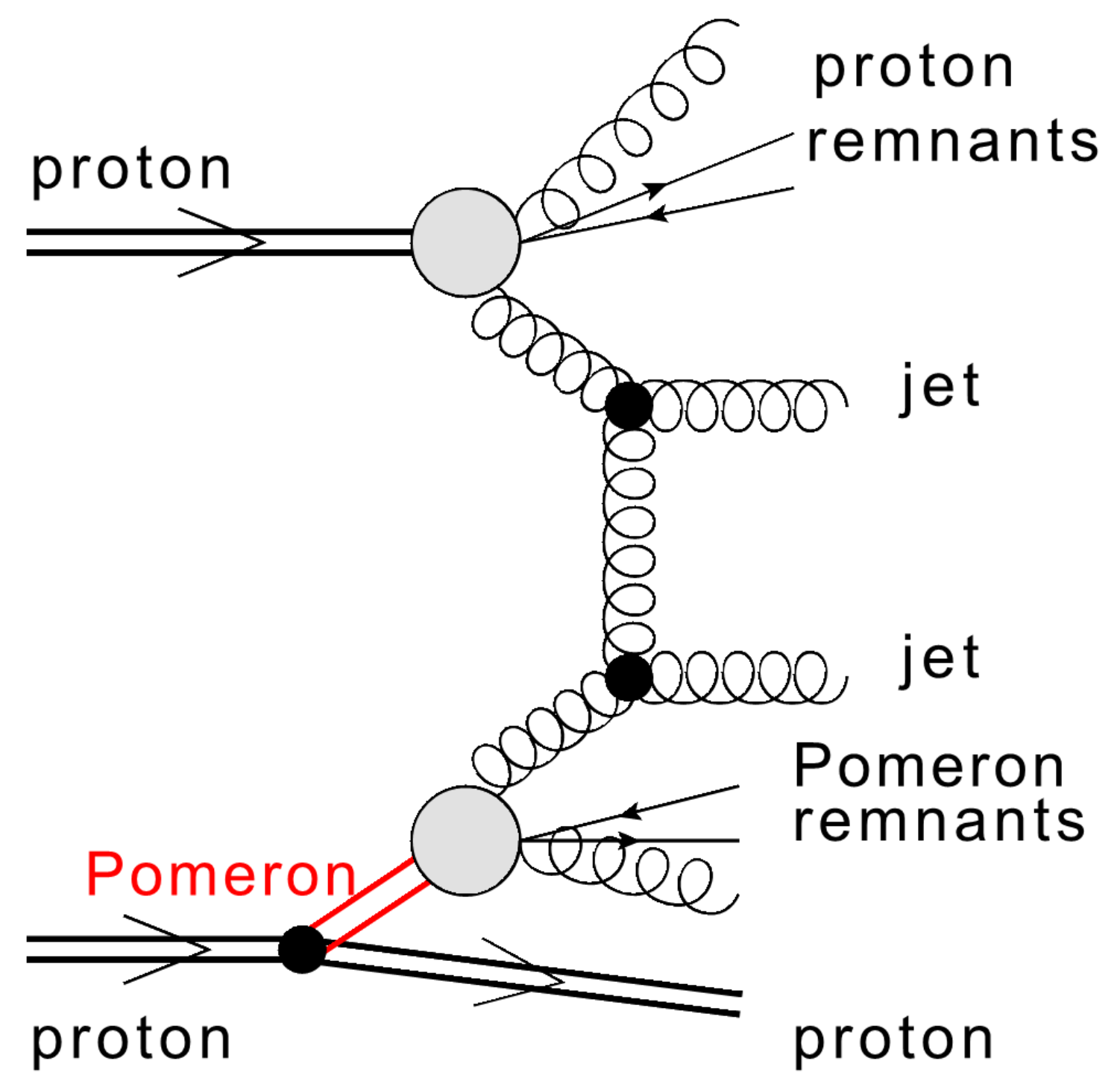




\section{Diffractive Dijet Production at 7 TeV, PLB 754 (2016) 214-234}

- Motivation

- Diffractive DIS at HERA: Diffractive parton densities dominated by gluon

- pp(pbar) collisions:

Failure in comparison of Tevatron

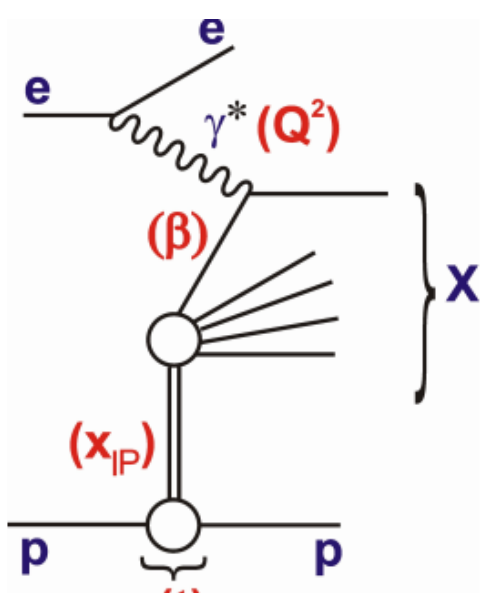

proton-tagged diffractive dijets with HERA DPDFs

-> 'rapidity gap survival probability' due to rescattering (absorptive corrections) breaks factorisation
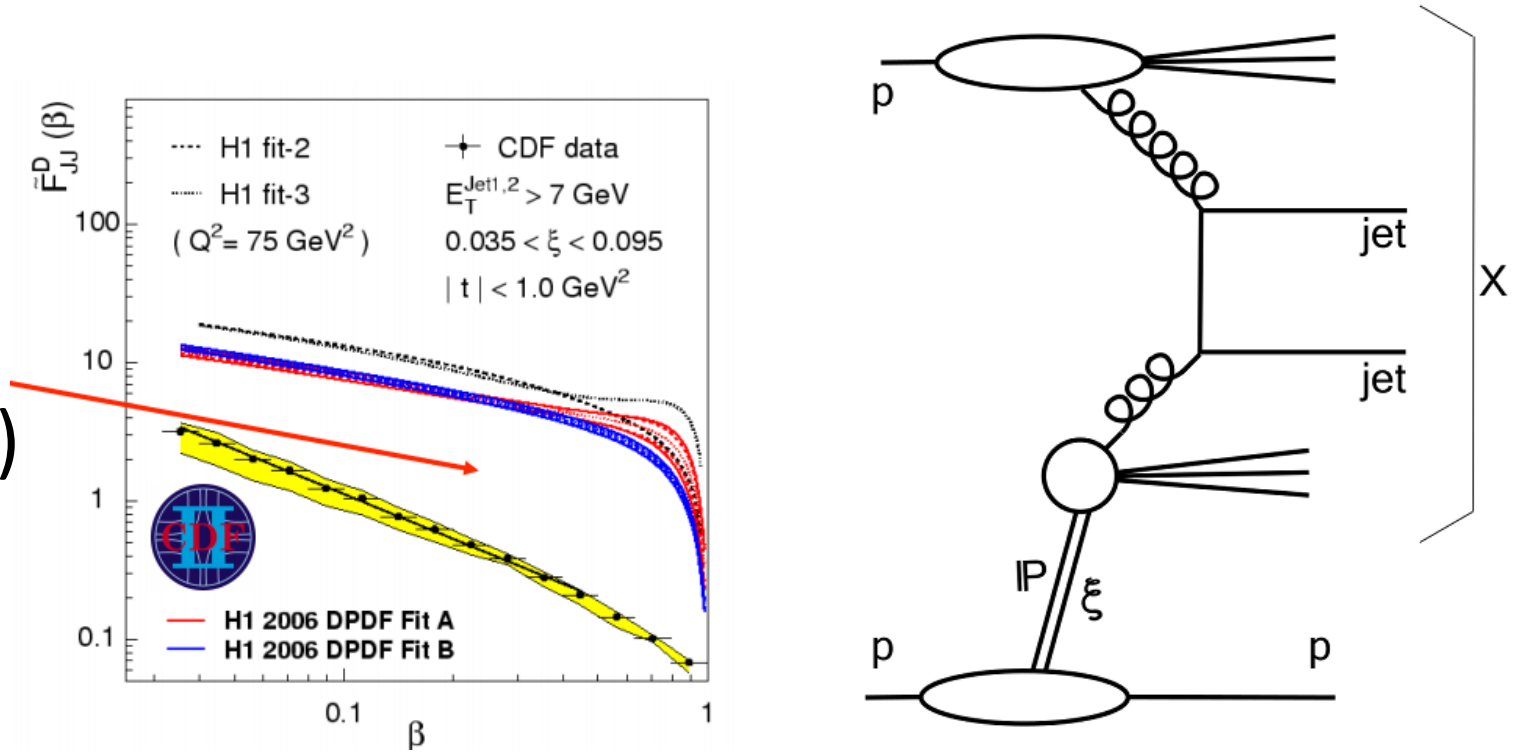


\section{Diffractive Dijet Production at 7 TeV, PLB 754 (2016) 214-234}

\section{- Kinematics and selection}

- Low pile-up data sample from 2010 with $v s=7 \mathrm{TeV}$ and integrated luminosity of $6.8 \mathrm{nb}$

- Jets with anti-kT algorithm, pT $>20 \mathrm{GeV},|\eta|<4.4, \mathrm{R}=0.4,0.6$

- Gaps characterisedusing $\Delta \eta_{F}$, based on tracks $(|\eta|<2.5, p T>200$ $\mathrm{MeV})$ and calocells $(|\eta|<4.8)$ that are $>5 \sigma$ out of noise distribution

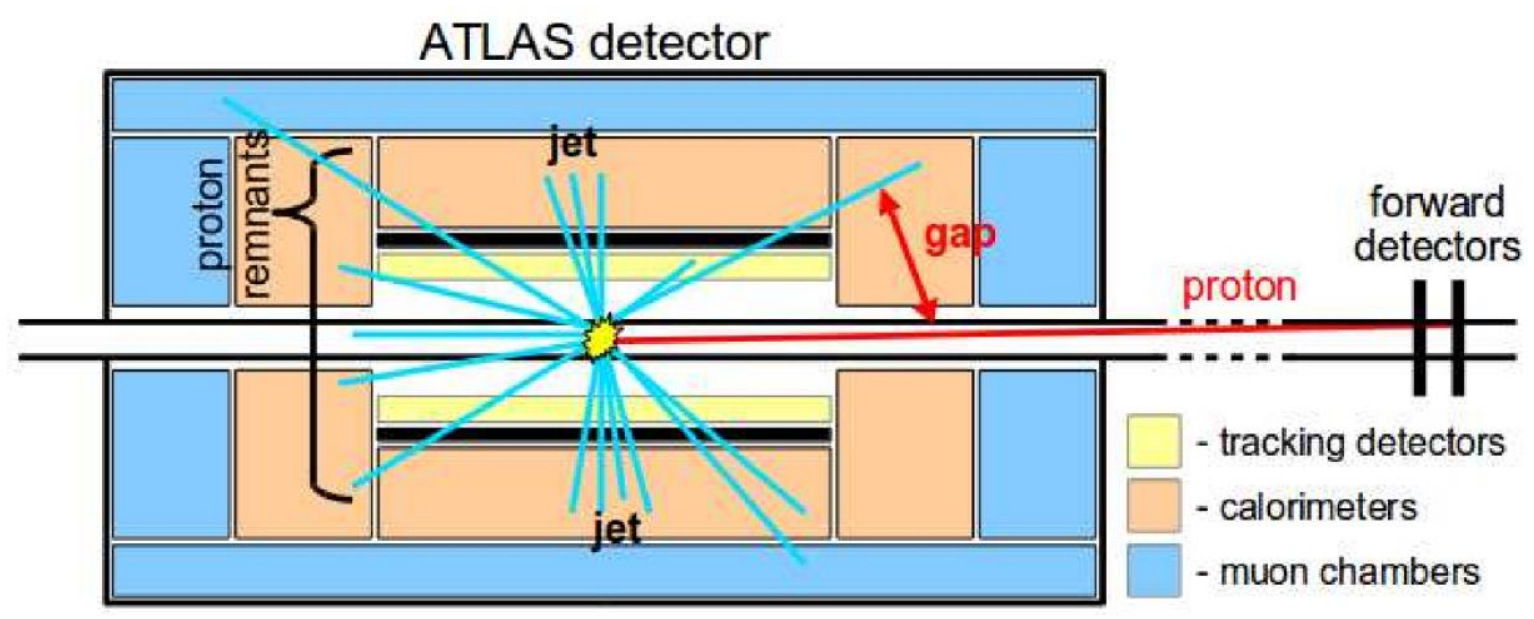




\section{Diffractive Dijet Production at 7 TeV, PLB 754 (2016) 214-234}

\section{- Event characteristics}

- Diffractive proton energy loss $(\xi)$ is extracted from energy deposits:

$$
\tilde{\xi} \simeq M_{\mathrm{X}}^{2} / s=\sum p_{\mathrm{T}} e^{ \pm \eta} / \sqrt{s}
$$

- Experimental resolution on $\log (\xi)$ is approximately $10 \%$
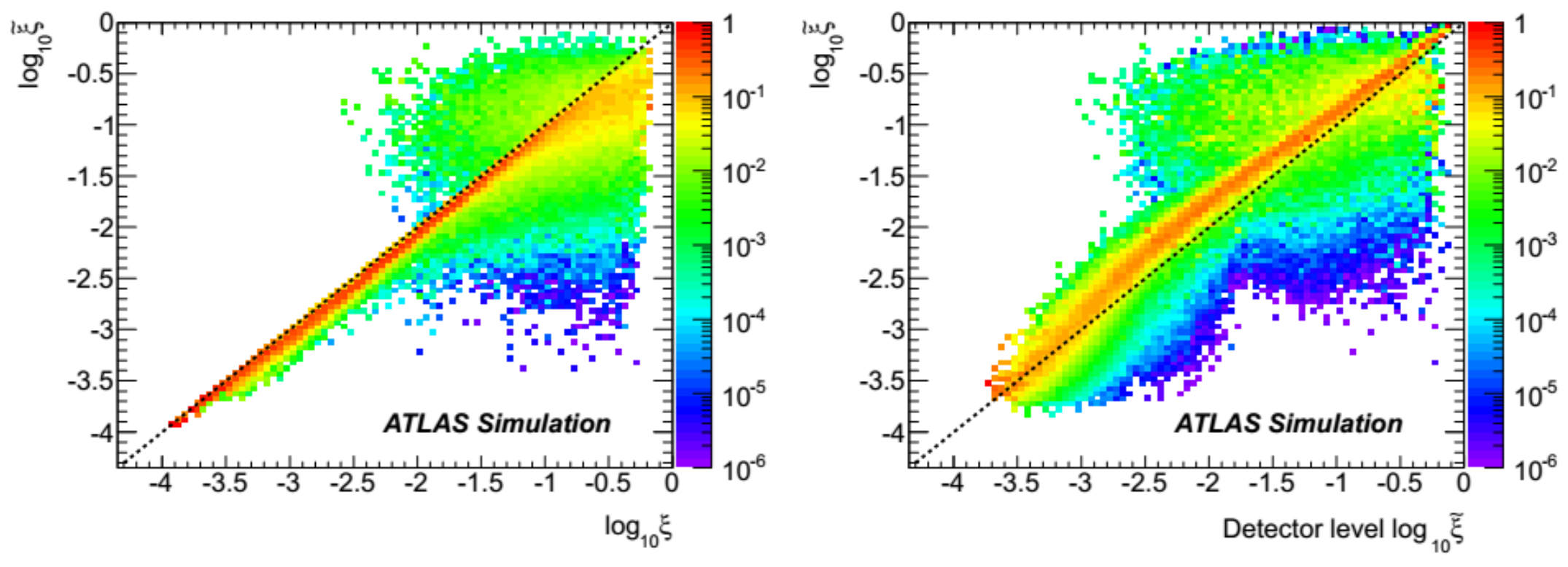


\section{Diffractive Dijet Production at 7 TeV, PLB 754 (2016) 214-234}

- Results

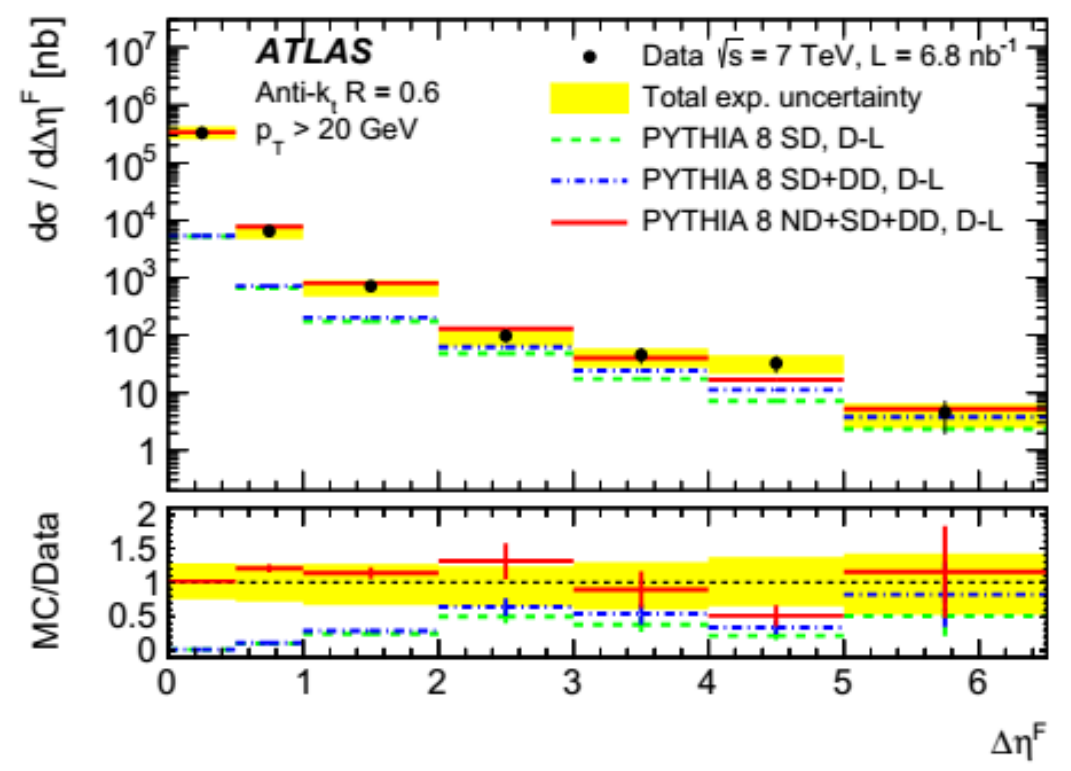

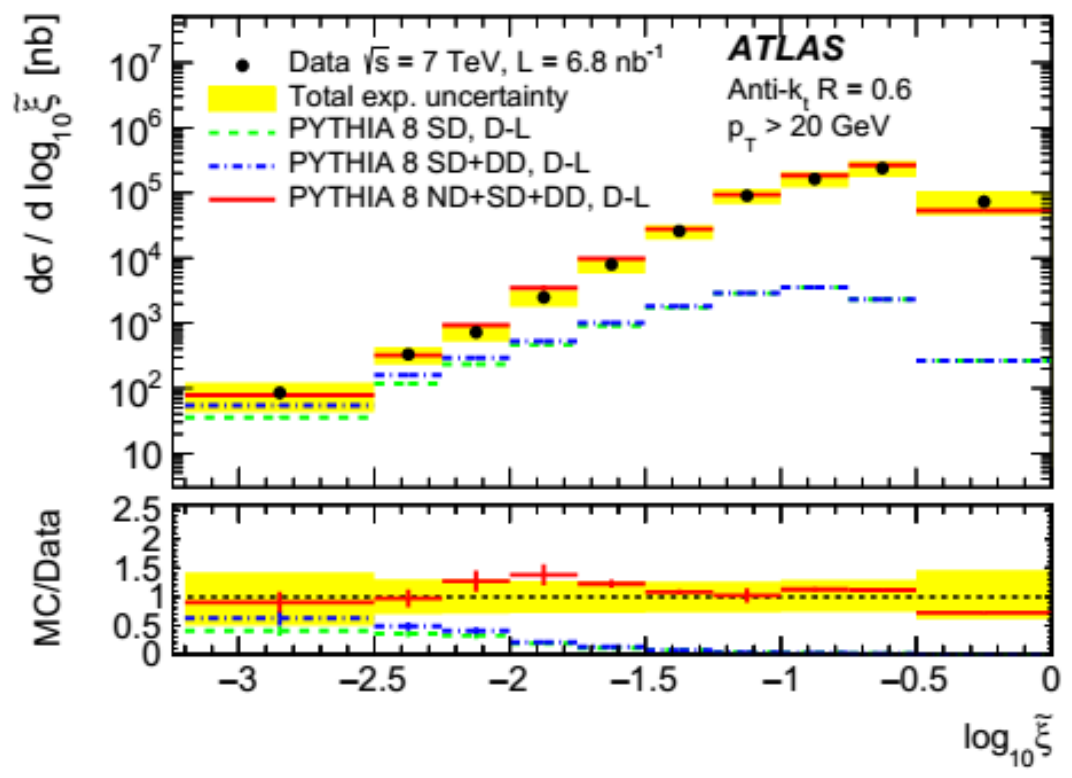

- Diffractive component is required for more complete description of data

- Pythia8 gives a good description of shape and normalization

- Rapidity gap survival factor is extracted in the context of POMWIG (and H1 2006 Fit B DPDFs):

$$
S^{2}=0.16 \pm 0.04 \text { (stat.) } \pm 0.08 \text { (exp. syst.) }
$$


proton

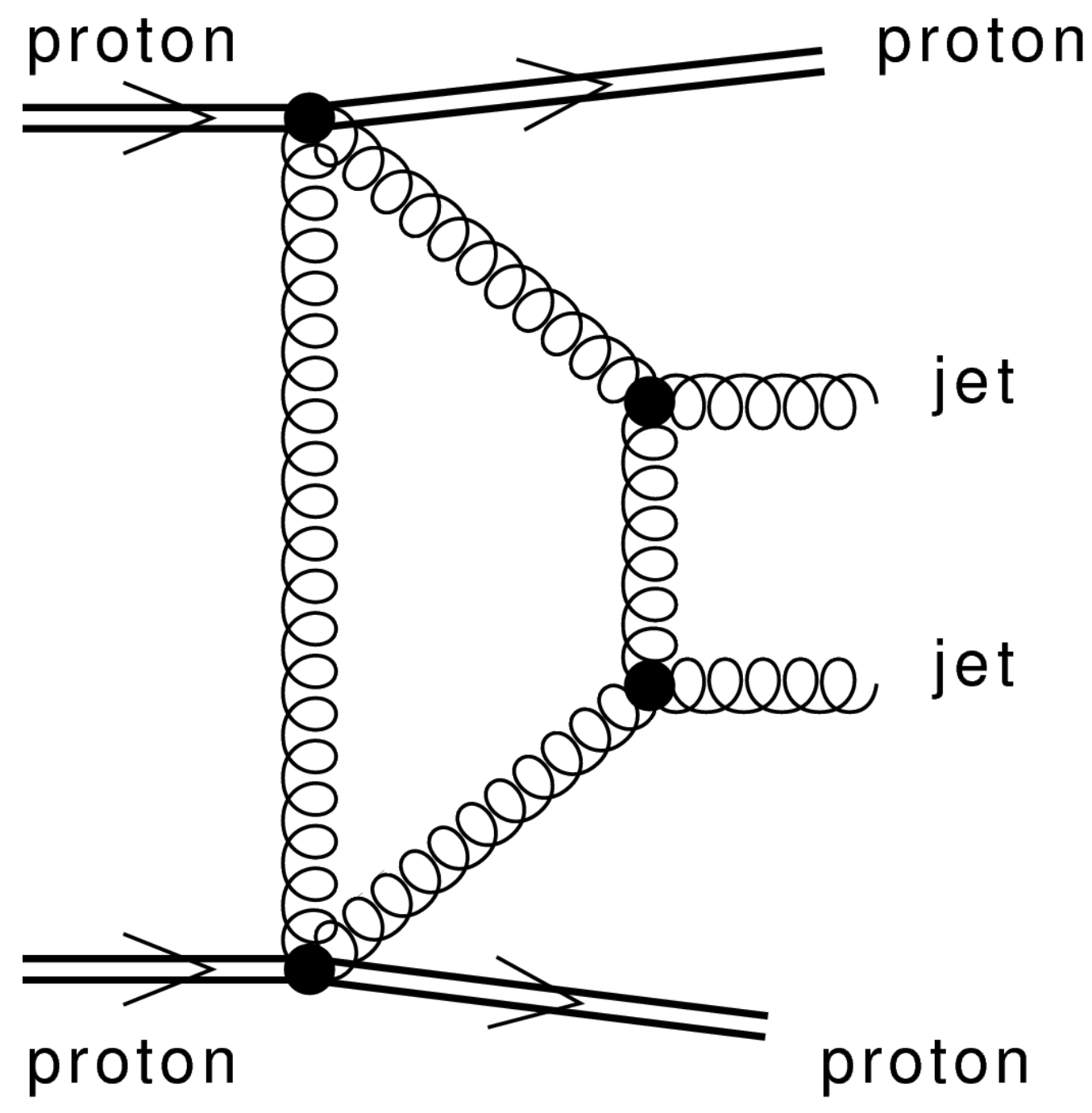


Feasibility Studies for Exclusive Jet Production with AFP, ATL-PHYS-PUB-2015-003

- AFP detector status (see Marek's talk)

- Single-arm with 3 3D pixel detector layers (near station) and $\mathbf{4}$ layers (far station) fully integrated with ATLAS

- 300 b fill \#4906 (10th of May 2016), AFP readout ( $20 \sigma$ from the beam) but triggered by ATLAS ( $\mu \lesssim 26,2: 16$ hrs)

- Low- $\mu$ run with dedicated AFP-based triggers is also recently recorded $\left(\approx 0.04 \mathrm{pb}^{-1}\right)$

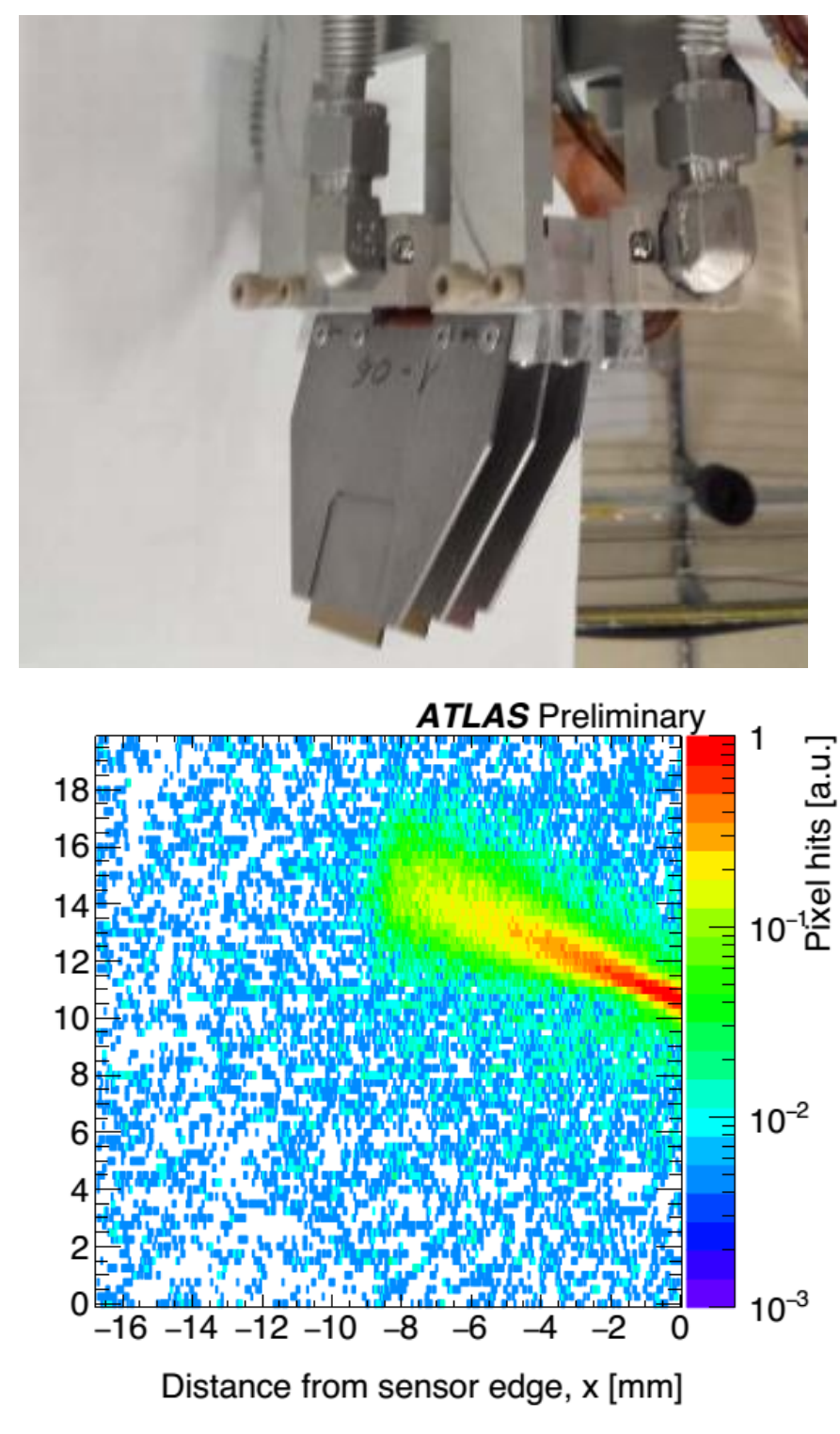




\section{- Motivation and feasibility results}

- Constrains other exclusive productions (e.g. Higgs)

- Cross section measurement is possible, even with single-tag configuration:

- $\mathrm{S} / \mathrm{B}=10^{4}$ after applying all the selection requirements

- 400 events expected with $1 \mathrm{pb}^{-1}$ of data

- See also EPJC 75 (2015) 320
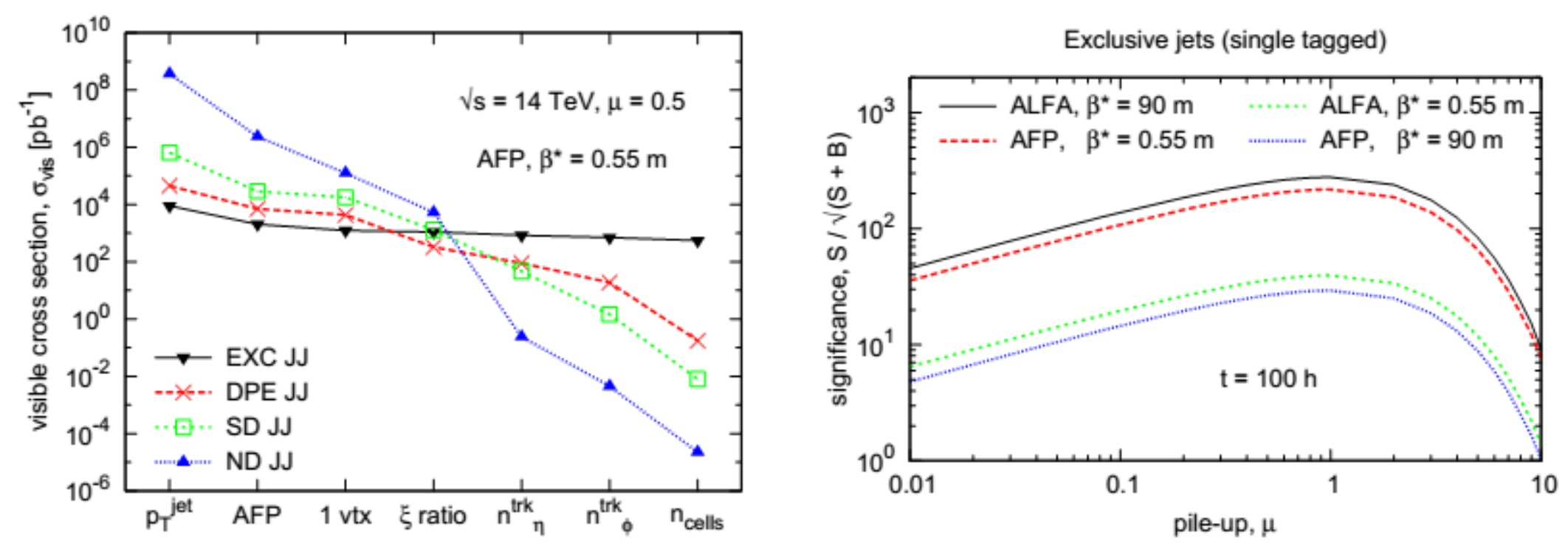


\section{Summary}

- Exclusive (photon-induced) processes

- Cross sections of the exclusive $\gamma \gamma \rightarrow \ell^{+} \ell^{-}$production have been measured -> Observation is consistent with the suppression (20\%) expected due to proton absorption contributions

- Evidence of SM exclusive $\gamma \gamma \rightarrow W^{+} W^{-}$production (significance of $3 \sigma$ ) $\rightarrow$ No evidence for an excess in the kinematic region targetting aQGC -> Limits on exclusive Higgs production cross section are also set

- Diffractive Dijets

- Evidence for diffractive contribution in $7 \mathrm{TeV}$ data

- Detailed understanding heavily limited by poorly known non-diffractive contribution

- Future prospects with dedicated proton spectrometers (AFP) are very promising 